\title{
Anti-feministische Mobilisierung in Europa. Kampf um eine neue politische Hegemonie?
}

\section{Birgit Sauer}

Online publiziert: 4. September 2019

(C) Der/die Autor(en) 2019

Zusammenfassung In der vergangenen Dekade formierte sich in Europa, aber auch in den USA und in Russland, eine anti-feministische Bewegung, die unter der selbstgewählten Bezeichnung „Anti-Genderismus“ gegen Gleichstellungspolitik (Gender Mainstreaming), Frauen- und Geschlechterforschung (Gender Studies) sowie gegen sexuelle Differenz (z.B. Homo-Ehe) mobilisiert. In jüngster Zeit haben auch rechtspopulistische und extrem rechte Akteure den Anti-Gender-Diskurs für ihre nativistische und völkische Propaganda entdeckt. Der Artikel leuchtet die gesellschaftlichen und politischen Ursachen der anti-genderistischen Mobilisierung aus und verortet deren Erfolg in den umfassenden Transformationen der Geschlechterregime, vor allem in sogenannten starken Familienernährerstaaten wie Deutschland und Österreich. Der Text argumentiert, dass Geschlechter- und Sexualitätsverhältnisse eine prominente Rolle in einem neuartigen Kampf um politische Hegemonie einnehmen, wenn auch mit unterschiedlichem Tempo und unterschiedlicher thematischer Schwerpunktsetzung in den europäischen Ländern. Mit einer Frame-Analyse ausgewählter Texte der Anti-Gender-Akteure arbeitet der Artikel zentrale Argumentationsmuster heraus und zeigt, dass „Gender“ im rechtspopulistischen Diskurs als ,leerer Signifikant“ wirkt: Ganz unterschiedliche Themen der antagonistischen rechten Kommunikation können mit bzw. gegen „Gender“ aufgerufen und in eine Strategie gegen „die da oben“ sowie gegen die vermeintlich „Anderen“ eingebaut werden. So bildet der Anti-Gender-Diskurs die Grundlage einer ,männlichen Identitätspolitik“.

Schlüsselwörter Anti-Genderismus · Rechtspopulismus · Männliche Identitätspolitik · Geschlechterverhältnisse

Prof. Dr. B. Sauer $(\bowtie)$

Institut für Politikwissenschaft, Universität Wien, Universitätsstraße 7, 1010 Wien, Österreich

E-Mail: birgit.sauer@univie.ac.at 


\title{
Anti-feminist mobilization in Europe. Struggle for a new political hegemony?
}

\begin{abstract}
Over the past decade an anti-feminist movement has emerged not only in Europe, but also in the United States and Russia. Under the self-chosen label "Anti-Genderism", the movement has mobilized against gender equality policies (gender mainstreaming), gender and women's studies, and sexual difference (e.g. homosexual marriage). Recently, right-wing populist and extreme right-wing actors have also discovered the anti-gender discourse for the purposes of their own nativist and nationalistic propaganda. The article sheds light on the social and political causes of anti-genderist mobilization and locates its success in the comprehensive transformations of gender regimes, especially in so-called strong male breadwinner states such as Germany and Austria. The text argues that gender and sexuality play a prominent role in a new battle for political hegemony, albeit at different speeds and with varying thematic priorities across European countries. Utilizing a critical frame analysis of selected texts from the anti-gender actors, the article elaborates central arguments and shows that "gender" in the right-wing populist discourse works as an "empty signifier": very different topics of antagonistic right communication can be invoked with or against "gender" and incorporated into a strategy against "the privileged elites" and against the supposed "others". In this way the anti-gender discourse forms the basis of a "male identity politics".
\end{abstract}

Keywords Anti-genderism $\cdot$ Right-wing populism $\cdot$ Male identity politics $\cdot$ Gender relations

\section{Anti-feministische Bewegungen als Problem. Einleitung}

In der vergangenen Dekade formierte sich in Europa, aber auch in den USA und in Russland, eine anti-feministische Bewegung, die unter der selbstgewählten Bezeichnung „Anti-Genderismus“ gegen Gleichstellungspolitik (Gender Mainstreaming), Frauen- und Geschlechterforschung an Universitäten (Gender Studies) sowie gegen sexuelle Differenz, sei es die Homo-Ehe oder Sexualerziehung an Kindergärten und Grundschulen, mobilisiert. Zu dieser Bewegung zählen die Proteste La Manif Pour Tous gegen die „Ehe für alle“ in Frankreich seit dem Jahr 2013 sowie die deutschen ,Demos für Alle“, die sich am französischen Vorbild orientieren (Blum 2015). In Spanien und Italien fanden bereits 2005 und 2007 anti-genderistische Großdemonstrationen mit ähnlicher Stoßrichtung statt (Paternotte 2015). Daher wird die Anti-Gender-Mobilisierung als Reaktion auf die Erfolge von schwulen und lesbischen Bewegungen bzw. der Frauengleichstellung gesehen. Doch ist die Bewegung vor allem auch in jenen Ländern erfolgreich, wo der Prozess der Gleichstellung von Frauen und der Emanzipation von LGBTIQ-Personen ${ }^{1}$ weit fragiler ist, wie Elżbieta Korolczuk am Beispiel Polens ausführt (Korolczuk 2015, S. 52). Polnische und slowakische Bischöfe organisierten beispielsweise Vorträge gegen Gender

\footnotetext{
${ }^{1}$ LGBTIQ steht für lesbisch, schwul (gay), bisexuell, trans- und intersexuell sowie queer.
} 
und veröffentlichten im Dezember 2013 einschlägige Hirtenbriefe (Marschütz 2014; Mad'arova 2015). In anderen europäischen Ländern agiert die katholische Kirche zurückhaltender und säkularisiert ihre Argumentationsstrategien gleichsam auf der Basis (natur-)wissenschaftlicher Gesichtspunkte, wie in Slowenien, Kroatien oder Frankreich (Kuhar 2015; Stambolis-Ruhstorfer und Tricou 2017). Neben der katholischen Kirche, die global gegen Gender agitiert, sind auch evangelikale Gruppen oder Teile der russisch-orthodoxen Kirche an der Anti-Gender-Front beteiligt.

In jüngster Zeit haben auch rechtspopulistische und extrem rechte Akteure den Anti-Gender-Diskurs für ihre nativistische und völkische Propaganda entdeckt. Das erste Positionspapier der „Pegida“ vom Dezember 2014 kritisierte beispielsweise in zwei seiner 19 Punkte sexuelle Selbstbestimmung und Gender Mainstreaming. ${ }^{2}$ Auch das Wahlprogramm der Alternative für Deutschland AfD (2013) reflektiert diesen anti-feministischen Impetus, und die Regierung Orban setzte als Vorreiterin des europäischen „Anti-Genderismus“ die Abschaffung des Faches Gender Studies an ungarischen Universitäten durch.

Das Konzept „Gender“, das von diesen diversen Gruppierungen aufgegriffen und vor allem heftig angegriffen wird, wird teilweise bewusst missverstanden und missinterpretiert, um in einem anti-feministischen Gestus die Einsichten der Frauenund Geschlechterforschung zu verzerren und lächerlich zu machen oder Maßnahmen der Gleichstellungspolitik zu delegitimieren (Marschütz 2014). So wird von der „Abschaffung“ von Geschlecht bzw. einer Aufforderung zu einer individuellen, bedingungslosen freien Wahl des eigenen Geschlechts gesprochen: Beispielhaft ist hier der Buchtitel der österreichischen FPÖ-Politikerin Barbara Rosenkranz (2008) MenschInnen. Gender-Mainstreaming. Auf dem Weg zum geschlechtslosen Menschen. Allerdings zeigt die Mobilisierung gegen Gender auch, dass die Akteure den Kern der wissenschaftlichen Erkenntnisse und politischen Prämissen, die mit dem GenderKonzept verknüpft sind, durchaus verstanden haben: Es geht darum, was die zweite Frauenbewegung seit Simone de Beauvoir (damals war das Konzept „Gender“ freilich noch nicht eingeführt) proklamiert: „Man ist nicht als Frau geboren, man wird es" (Beauvoir 1992, S. 334). In den Worten Sabine Harks und Paula-Irene Villas scheint den selbsternannten Anti-Genderisten klar, ,dass der Begriff auf die - im weitesten Sinn - soziale Beschaffenheit von Geschlecht zielt und damit eine naive simplifizierende Vorstellung von Geschlecht als naturhafte, unveränderliche, an-sich-so-seiende Tatsache jenseits sozialer, kultureller und spezifisch historischer Bedingtheiten überwindet" (Hark und Villa 2015, S. 7).

Mein Artikel will die Frage beantworten, warum der Kampf gegen Gender europaweit vergleichsweise erfolgreich wurde. Um diese Frage beantworten zu können, müssen die gesellschaftlichen und politischen Ursachen der anti-feministischen Mobilisierung unter dem Label „Anti-Genderismus“ ausgeleuchtet werden. Die AntiGender-Mobilisierungen, so die These meines Textes, müssen als Ergebnisse umfassender Transformationen europäischer Gesellschaften, vor allem der Geschlechterregime in sogenannten starken Familienernährerstaaten wie Deutschland und Österreich (Langan und Ostner 1991) verortet werden. Geschlechter- und Sexualitätsverhältnisse, so mein Argument weiter, nehmen eine prominente Rolle in einem

\footnotetext{
2 http://www.i-finger.de/pegida-positionspapier.pdf, letzter Zugriff 15.06.2016.
} 
neuartigen Kampf um Hegemonie ein - wenn auch mit unterschiedlicher Geschwindigkeit und unterschiedlicher Deutlichkeit in europäischen Ländern. Der Kampf gegen „Gender“ ist, so will ich deutlich machen, Einsatz in einem umfassenden Kampf um die Transformation europäischer Gesellschaften, um politische Macht und um Hegemonie (Laclau und Mouffe 2001, S. 129). In den Auseinandersetzungen um „Gender“ werden also nicht allein Geschlechter- und Sexualitätsverhältnisse, sondern auch Vorstellungen von Demokratie, vom Verhältnis zwischen Staat und BürgerInnen in Frage gestellt und neu verhandelt. „Gender“ fungiert in diesen Auseinandersetzungen als „leerer Signifikant“ (Laclau 1996, S. 36), der eine ganze Kette von Bedeutungen und Verhältnissen, die kritisiert und verändert werden sollen, aufruft.

Im ersten Schritt werde ich zentrale Akteure des „Anti-Genderismus“ vorstellen und dann im zweiten Schritt deren wichtigste Argumentationsstränge herausarbeiten. Im Anschluss daran skizziere ich die Veränderungen europäischer Gesellschaften, um zu erklären, dass und wie „Gender“ als ein ,leerer Signifikant“ in einem Hegemonialprojekt funktioniert, das zum einen männliche Identitätspolitik ist und zum anderen die Liberalisierung und Demokratisierung europäischer Gesellschaften seit den 1970er-Jahren angreift, ja, liberale Demokratie per se in Frage stellt.

\section{Akteure des selbsternannten ,Anti-Genderismus“}

In ihrer heute gängigen, diffamierenden Verwendungsweise wurden die Begriffe „Gender-Theorie“ oder „Gender-Ideologie“ von der katholischen Kirche Mitte der 1990er-Jahre als Reaktion auf die Integration des Gender-Konzepts in die Abschlussdokumente der UN-Konferenzen in Kairo 1994 und Beijing 1995 geprägt (Marschütz 2014; Paternotte 2014, 2015; Favier 2015). Angriffspunkte waren damals vor allem reproduktive Rechte für Frauen, also das Recht auf Abtreibung, sowie die Anerkennung von Homosexualität. Beides verknüpften die katholischen Vertreter mit dem Gender-Konzept. Diese katholische Gegenposition zum Gender-Konzept wurde schließlich in den Folgejahren in einer Reihe kirchlicher Schreiben weiter ausformuliert wie beispielsweise im vom Päpstlichen Rat für die Familie im Jahr 2000 herausgegebenen Dokument Ehe, Familie und "faktische Lebensgemeinschaften “. Darin wird die vermeintliche Gefahr der konstruktivistischen „Gender-Ideologie“ folgendermaßen beschrieben:

Jede sexuelle Einstellung, und damit auch die Homosexualität, sei so zu rechtfertigen. Die Gesellschaft müsse sich ändern, um in der Gestaltung des Gesellschaftslebens anderen Geschlechtern neben dem männlichen und dem weiblichen Platz zu machen (Päpstlicher Rat für die Familie 2000).

In Europa avancierten seit der Millenniumswende der Vatikan, katholische Intellektuelle und katholische Laienorganisationen zu zentralen Akteuren der AntiGender-Mobilisierung. Auch transnationale christliche Akteursnetzwerke wie der „World Congress of Families“, der 1995 als US-amerikanisch-russisches Projekt gegründet wurde, befeuern diese globale anti-feministische Mobilisierung (Moss 2017). Eine der international prominenten Figuren, die in diesen Kontexten auftritt, 
ist die deutsche Publizistin Gabriele Kuby, deren 2006 erschienenes Buch GenderRevolution - Relativismus in Aktion (Kuby 2006) ebenso wie ihre späteren Veröffentlichungen wesentlich zur Popularisierung des Anti-Gender-Diskurses beitrugen, insbesondere in den Kirchen postkommunistischer Länder (Marschütz 2014; Chołuj 2015, S. 221; Korolczuk 2015, S. 47).

Neben Protestveranstaltungen im öffentlichen Raum gehört zum Mobilisierungsrepertoire der Anti-Gender-Gruppierungen auch die Nutzung digitaler Formen der mehrsprachigen konservativen Online-Petitionsplattform CitizenGo (Hodžić und Bijelić 2014, S. 11; vgl. auch Korolczuk 2015, S. 47). CitizenGo organisierte beispielsweise die Kampagne gegen den Estrela-Report - den Bericht über sexuelle und reproduktive Gesundheit und die damit verbundenen Rechte (Ausschuss für die Rechte der Frau und die Gleichstellung der Geschlechter 2013), der u.a. einen legalen und sicheren Zugang zu Schwangerschaftsabbrüchen forderte. Die wenige Monate später organisierte Online-Kampagne gegen den Lunacek-Report (EU-Fahrplan zur Bekämpfung von Homophobie und Diskriminierung aufgrund von sexueller Orientierung und Geschlechtsidentität, Ausschuss für bürgerliche Freiheiten, Justiz und Inneres 2014) konnte die Annahme des Berichts Anfang Februar 2014 allerdings eben so wenig verhindern wie den Estrela-Report (Hodžić und Bijelić 2014, S. $18 \mathrm{f}$.).

In Deutschland und Österreich haben sich zudem Gruppen sogenannter „,besorgter Eltern“ zusammengeschlossen, die vor der vermeintlichen „Frühsexualisierung“ durch Sexualerziehung in Kindergärten und Schulen warnen (Schmincke 2015). Der katholische Diskurs der „Gender-Ideologie“ fand in einigen europäischen Ländern Widerhall bei sogenannten Männer- und Väterrechtsbewegungen (Weiss 2013). Diese kritisieren nicht allein Gleichstellungspolitik, die sie diskriminiere und ihrer Rechte (z. B. auf ihre Kinder) beraube, sondern darüber hinaus eine generelle „Verweiblichung“ der Gesellschaft. Mit der Rede von einer angeblichen „Krise der Männlichkeit" geht ein aggressiver Maskulinismus einher, wie das Zitat des AfDPolitikers Björn Höcke, der den Topos der Feminisierung aufgreift, zeigt: ,,Wir müssen unsere Männlichkeit wiederentdecken. Denn nur wenn wir unsere Männlichkeit wiederentdecken, werden wir mannhaft. Und nur wenn wir mannhaft werden, werden wir wehrhaft, und wir müssen wehrhaft werden, liebe Freunde! “3

Diese Einlassungen zeigen, dass rechtspopulistische und rechtsextreme Gruppierungen den sogenannten „Anti-Genderismus“ vorantreiben. In Österreich kam die Debatte um die „Gender-Ideologie“ erstmals 2008 durch eine Publikation der deutschnationalen, nicht-religiösen FPÖ-Politikerin Barbara Rosenkranz (2008) auf die Agenda. Auch in Deutschland vertreten die Parteien AfD und NPD ebenso wie Pegida Anti-Gender-Argumente (Blum 2015; Ganz 2015; Lang 2015) sowie die Identitäre Bewegung und deren sich als feministisch bezeichnender Ableger „120 Dezibel“", eine Anti-Gender-Haltung.

In dieser rechtspopulistischen bzw. extrem rechten Variante findet der „AntiGenderismus" seinen Hintergrund in einer völkisch-rassistischen Sorge um die demographische Entwicklung europäischer Staaten (unter Bezug auf Renaud Camus'

\footnotetext{
${ }^{3}$ Björn Höcke auf dem Parteitag der Alternative für Deutschland im November 2015 in Erfurt, https:// www.youtube.com/watch?v=yBvy0MR3KBE (abgerufen am 30.11.2016).
} 
(2016) Konzept des ,großen Austauschs“), die mit anti-muslimischen Argumentationsmustern gegen die Flüchtlingsbewegungen verzahnt wird (Mayer et al. 2015).

Dem „Anti-Genderismus“ gelingt es also, eine große Bandbreite recht unterschiedlicher Akteure zusammenzufügen und partielle Übereinstimmungen zwischen konservativen katholischen Gruppierungen, äußerst elitären, ultrakonservativen Organisationen wie etwa dem internationalen rechts-katholisch und auf ein adliges Klientel ausgerichteten Verband „Tradition, Familie, Privateigentum“ und rechtspopulistischen Gruppen, die sich eher als Vertretung der „kleinen Leute“ inszenieren, wie auch Gruppen aus dem links-liberalen Milieu, die gegen die ,political correctness“ von „Gender“ polemisieren (Pfaller 2017), zu schaffen.

\section{Zentrale Deutungsmuster der Anti-Gender-Mobilisierung}

Im Folgenden möchte ich sechs zentrale Argumentationsmuster skizzieren, die im europäischen Kontext gegen „Gender“ aufgerufen werden. Als erstes fällt das Beharren auf einer ,natürlichen“ Zweigeschlechtlichkeit auf, die mit der Vorstellung von traditionellen Frauen- und Männerbildern und vor allem einer patriarchalen geschlechtsspezifischen Arbeitsteilung einhergeht. Zwar hat sich die politische Rechte in den letzten Jahren rhetorisch modernisiert und schließt nun an Gleichstellungsdiskurse an. Amesberger und Halbmayr (2002, S. 308) konstatieren für die österreichische Freiheitliche Partei (FPÖ) beispielsweise ein ,modernisiertes traditionelles Rollenbild“, wie dies de Lange und Mügge (2015, S. 63) auch für Belgien und die Niederlande feststellen konnten. Dennoch gehört die Berufung auf ein zweigeschlechtlich-polares Geschlechterkonzept, auf die traditionelle geschlechtsspezifische Arbeitsteilung und damit verbundene Geschlechterrollen des männlichen Familienernährers und der Frau als Mutter und Sorgearbeiterin noch immer zum rechten populistischen Repertoire in Europa (Sauer et al. 2017, S. 113).

Eine zweite zentrale Argumentationsfigur, die sich in unterschiedlichen nationalen und lokalen Kontexten artikuliert, fokussiert auf den „Schutz der Familie“, namentlich der naturalisierten, heterosexuellen Kleinfamilie (Schmincke 2015), die durch „Gender“ als gefährdet erscheint. Den einen „Anti-Genderisten“ gilt die Familie als „Keimzelle“ des Staates, der Gesellschaft oder der Nation, den anderen als Verkörperung christlich-konservativer Werte und wieder anderen wie etwa der AfD als Versinnbildlichung ,unsere(r) gemeinsame(n) Zukunft“ (AfD 2013) oder im Kontext anderer rechtsextremer Weltdeutungen als völkisches Bollwerk.

Drittens ist mit der Anrufung der Figur des bedrohten Kindes eine homophobe Argumentation verbunden, besonders dann, wenn gegen Sexualerziehung in öffentlichen Einrichtungen agitiert wird. Dann werden zudem Elternrechte in Bezug auf die Erziehung von Kindern eingefordert, so dass über die „Chiffre Kind“ (Schminke 2015) auch das Verhältnis von Staat und privatem Bereich neu verhandelt und einer Re-Privatisierung von Erziehung das Wort geredet wird.

Viertens wird dem „Genderismus“ vorgeworfen, die BürgerInnen zu bevormunden, ja einen neuen Totalitarismus zu befördern. Häufig wird diese Unterstellung an inter- bzw. supranationale Organisationen - die EU oder die UNO - im Stile einer populistischen Anti-Elite-Argumentation adressiert, oft aber auch gegen nur 
vage definierte Eliten „der Genderisten“ gerichtet, seien dies sozialdemokratische Gleichstellungspolitikerinnen oder Gender-ProfessorInnen. Die „Gender-Ideologie“ wolle in totalitärer Weise die Gesellschaft umgestalten und beabsichtige, das Leben jedes einzelnen zu dominieren. Dies kommt zum Teil als anti-europäische Haltung (besonders in Russland; vgl. Moss 2017), als Anti-EU-Rhetorik (in europäischen rechtspopulistischen Diskursen; vgl. Mayer und Sauer 2017), als Anti-Amerikanismus (Ganz 2015) oder als Wiederkehr eines totalitären Sozialismus (vor allem, aber nicht nur in post-kommunistischen Staaten; Graff 2014, S. 433) daher. Die Wortkreation „Gender-Ideologie“ verweist in diesem Deutungsmuster auf ein dem Alltagsdiskurs entlehntes Verständnis von Ideologie - nämlich falsches Bewusstsein mit dem Zweck der Manipulation.

Ein fünftes Deutungsmuster ist die Konstruktion eines vermeintlich gleichberechtigten und emanzipierten Okzidents und eines frauenunterdrückenden, intoleranten Orients. In dieser Figur wird Gleichberechtigung von Frau und Mann als europäischer Wert zur Abgrenzung gegenüber den „Anderen“ in Stellung gebracht, obgleich der „Anti-Genderismus“ traditionelle Geschlechterverhältnisse in europäischen Ländern wiederhergestellt sehen möchte. Die Verknüpfung von Anti-Gender- und AntiMigrations-Mobilisierung erklärt diese auf den ersten Blick widersprüchliche Konfiguration insbesondere rechter Akteure: Das Beharren auf die „Frauenfreundlichkeit“ und die Gleichstellungskultur europäischer Gesellschaften entpuppt sich als Legitimation für den Ausschluss der als „Andere“ identifizierten Menschen, wenn etwa Gewalt gegen Frauen ausschließlich in externalisierter Form auf Geflüchtete und Migranten projiziert wird (Dietze 2015). Sara Farris (2017) hat diese Argumentation als „Femonationalismus“ bezeichnet, also die Aufwertung der eigenen Gesellschaft als „feministisch“ mit dem Ziel der Abwertung von „Anderen“.

Ein sechster Diskursstrang ist der Anti-Intellektualismus, wie er vor allem in rechtspopulistischen Argumentationslogiken zu finden ist. Angegriffen werden politische und intellektuelle Eliten von GleichstellungspolitikerInnen und ProfessorInnen der Gender Studies, die mit dem Gender-Begriff ein für die Mehrheit der BürgerInnen unverständliches Konzept entwickelt hätten, das zudem dem common sense völlig widerspreche.

Insgesamt wird in diesen sechs zentralen Deutungen ein Diskurs sichtbar, der den Verlust von einstigen Gewissheiten in den Geschlechterverhältnissen beklagt und die Aufrechterhaltung oder Re-Etablierung patriarchal geprägter Geschlechterverhältnisse einfordert. Zudem bedient der „Anti-Genderismus“ einen doppelten Antagonismus rechtspopulistischer Mobilisierung (Mudde und Kaltwasser 2015), nämlich gegen „die da oben“, die Eliten, und gegen die „Anderen“, die nicht Zugehörigen, und formt damit diskursiv ein ,natürliches“ Volk - die „,normalen Männer und Frauen“, der „,kleine Mann auf der Straße“. Allerdings muss auf die Breite und Varianz der Positionen ,anti-genderistischer“ Akteure verwiesen werden: Während sich u.a. im deutschsprachigen Raum, wo rechtspopulistische und rechtsextreme Kräfte zu wesentlichen TrägerInnen des Diskurses zählen, die Koppelung mit einer Anti-Migrations-Rhetorik häufig nachweisen lässt, lassen sich in Frankreich auch Versuche beobachten, mit konservativen Positionen in Bezug auf Gender-Politiken gerade migrantische Arbeiterfamilien als WählerInnen zu gewinnen (Brustier 2015). In skandinavischen Ländern korrelieren Anti-Gender-Diskurse eher mit wohlfahrts- 
chauvinistischen Vorstellungen, während Gleichstellungspolitiken nicht in Frage gestellt werden (Siim und Meret 2019).

\section{Soziale Veränderungen der Geschlechterverhältnisse. „Anti- Genderismus“" als männliche Identitätspolitik}

„Anti-Genderismus“ ist ohne Zweifel eine Reaktion auf die Erfolge feministischer, lesbisch und schwuler Bewegungen, die seit den 1960er-Jahren zu einer umfassenden Liberalisierung und Pluralisierung europäischer Gesellschaften beigetragen haben. Darüber hinaus wird der „Anti-Genderismus“ in der Literatur auch als Reaktion auf umfassende gesellschaftliche neoliberale Veränderungen der letzten 20 Jahre begriffen, mit denen Deregulierung und Prekarisierung von Arbeit, der Abbau wohlfahrtsstaatlicher Sicherungen, stagnierende Umverteilung, seit der Finanzkrise deutliche Wohlfahrtsverluste und das Öffnen der Wohlstandsschere verbunden sind. Diese ökonomischen und sozialen Veränderungen ließen das Vertrauen in die Handlungsfähigkeit der politischen Eliten sowie in demokratische Institutionen und Verfahren erodieren (Wimbauer et al. 2015; Grzebalska 2016).

Die ökonomische Verunsicherungsthese ist allerdings für die Erklärung des Erfolgs der Anti-Gender-Mobilisierung allein nicht haltbar, nicht zuletzt deshalb, weil die Anti-Gender-Front nicht nur oder vornehmlich aus offensichtlich von sozialem Abstieg bedrohten gesellschaftlichen Gruppen besteht, sondern im Gegenteil gerade in links-liberalen intellektuellen Kreisen das Anti-political-correctness-Argument große Bedeutung erlangt. Neben Klassenverhältnissen müssen daher auch Geschlechterverhältnisse und deren Transformation zur Erklärung des Phänomens in Betracht gezogen werden, denn schließlich verweist die „Gender“-Obsession der Anti-Gender-Akteure auf Veränderungen der Geschlechterverhältnisse seit den 1970er-Jahren, die paradigmatisch für eine umfassende Destabilisierung betrachtet werden. Ein Blick auf die longue durée europäischer, insbesondere konservativer Wohlfahrtsstaaten (Esping-Andersen 1990) macht die fundamentalen Transformationen von Geschlechterregimen sichtbar. Die Phase der Demokratisierung und des Ausbaus wohlfahrtsstaatlicher Institutionen erlaubte seit den 1970er-Jahren die Politisierung von Geschlechterungleichheit und führte u. a. zur sukzessiven, wenn auch langsamen und nur partiellen Integration von Frauen in den Erwerbsarbeitsmarkt, in nationalstaatliche, bis dahin exklusiv männliche Wohlfahrtsinstitutionen und in androzentrische politische Institutionen. Dieser Integrationsprozess erfuhr durch das neoliberale Arrangement von Markt und Staat seit den 1990er-Jahren eine, wenn auch geschlechterambivalente, Beschleunigung. Frauen wurden nun verstärkt aus der Familie herausgeführt und - wenn auch in der Regel prekär - in den Erwerbsarbeitsmarkt integriert. Vor allem in sogenannten konservativen Wohlfahrtsstaaten wie Deutschland und Österreich wurde so allmählich das männliche Familienernährermodell mit einem vergleichsweise hohen männlichen Familienlohn zugunsten des Zwei-Ernährermodells abgelöst. Der neoliberale Umbau kapitalistischer Gesellschaften veränderte also hierarchische Geschlechterregime und tradierte heterosexuelle Lebensweisen: Die geschlechtsspezifische Arbeitsteilung innerhalb von Famili- 
en sowie die Idee der heterosexuellen Kleinfamilie wurden zugunsten pluralistischer Arrangements in Frage gestellt (Candeias 2016, S. 19).

Allerdings wurde ein Grundproblem kapitalistischer Gesellschaften auf diese Weise nicht gelöst, nämlich die Externalisierung der Sorgearbeit (für Kinder, Kranke und Alte), die noch immer unbezahlt von Frauen erbracht werden soll. Daher muss die Anrufung von Frauen als Erwerbstätige klassenspezifisch differenziert betrachtet werden: Gut ausgebildete Frauen können beruflich erfolgreich sein, sie werden durch Gleichstellungsmaßnahmen gefördert und können familiäre Sorgearbeit tendenziell zukaufen. Weniger gut ausgebildete oder migrierte Frauen werden demgegenüber in unsichere Arbeitsverhältnisse gezwungen, nicht zuletzt in der Sorgearbeit. Neoliberale Gleichstellung(-spolitik), die die weibliche Erwerbsintegration begleitet, entpuppt sich als ein Projekt, das zu einer neuen geschlechtsspezifischen Arbeitsteilung entlang der Linien Ethnizität und Klasse führte.

Der neoliberale Umbau europäischer Gesellschaften implizierte schließlich auch ambivalente Formen der Maskulinisierung. Hegemoniale Männlichkeit inszenierte sich in einer Hypermaskulinität des finanzialisierten Kapitalismus, z. B. in der Welt der Banker und Börsianer. Im Unterschied dazu wurden marginalisierte Männlichkeiten (zur Begrifflichkeit: Connell und Messerschmidt 2005), also z. B. weniger gut ausgebildete oder erwerbslose Männer, fundamental in Frage gestellt.

Insgesamt war sowohl die systematische Aushöhlung des Wohlstands der Erwerbstätigen, die Prekarisierung von Arbeit und eine steigende Erosion des männlichen Normalarbeitsmodells von einer stärkeren Erwerbsbeteiligung von Frauen sowie der Förderung von gut ausgebildeten Frauen und der Entstehung neuer Vorstellungen hegemonialer Männlichkeit begleitet. Diese Prozesse ließen eine brisante Mischung aus realer oder befürchteter sozialer Degradierung und aus Verlusten im Erwerbsbereich, aber zugleich einer nach wie vor existierenden Ideologie des Familienernährers entstehen, der viele Männer nicht entsprechen können. Sie wurden so gleichsam zu ,gescheiterte(n) Patriarchen“ (Radhakrishnan und Solari 2015, S. 788-791).

Anti-Gender-Akteure greifen neben der Politisierung ihrer spezifischen Agenden (wie Anti-Abtreibung, Privatisierung von Kindererziehung, Anti-Migration) diese männlichen Enttäuschungen und Ohnmachtsgefühle auf. Sie deuten diese Konstellationen der Verunsicherung in eine „Krise der Männlichkeit“ sowie in eine Wut gegen die „Anderen“ um. Michael Kimmel (2013) nennt die in den USA auf diese Weise konstruierte Gruppe ,,angry white men“, wütende weiße Männer.

Zugleich steckt in diesen diskursiven Gesten also ein Angebot der Selbstaffirmierung, der Selbstbestärkung (vermeintlich) marginalisierter Männlichkeit, bieten doch Anti-Gender-Anrufungen im neoliberalen Verunsicherungsdiskurs Anknüpfungspunkte für eine Re-Etablierung traditioneller Geschlechterkonstellationen und -hierarchien. Der Selbstaffirmierung dient u. a. die Ablehnung von Gleichstellungspolitik, die als Privilegierung von insbesondere gut ausgebildeten Frauen dargestellt wird. Auch in femonationalistischen Argumentationen kann sich unterworfene Männlichkeit als liberal, aufgeklärt und modern - in Abgrenzung von muslimischen Männern - entwerfen.

Der „Anti-Genderismus“ bedient, so mein Fazit, diskursiv diese Konstellation veränderter Geschlechterverhältnisse, sich auflösender traditioneller Geschlechter- 
identitäten bzw. neuartiger Erwartungen an Frauen- und Männerbilder. „Anti-Genderismus" kann daher als männliche Identitätspolitik begriffen werden. Auch die Anrufung des „kleinen Mannes auf der Straße“ ist Teil dieser maskulinistischen Identitätspolitik. So formiert der Anti-Gender-Diskurs zwar eine Gruppe unterworfener und marginalisierter Männlichkeiten, doch er enthält zugleich das Versprechen, dass eine charismatisch-maskulinisierte Führung (die auch eine Frau sein kann) diesen Gruppen neue (Selbst-)Sicherheit verschaffen kann. Vor allem femonationalistische Argumentationsfiguren taugen dazu, unterworfene Männlichkeit im Gestus des Schutzes von Frauen der Mehrheitsgesellschaft vor migrantischen Männern zu erhöhen.

\section{Geschlecht als leerer Signifikant. Ein demokratiepolitisches Fazit}

Jenseits der Alltagssprache angesiedelt und der breiten Öffentlichkeit meist nur in Umrissen bekannt, eignet sich der Begriff „Gender“ für die Aufladung mit unterschiedlichen Inhalten und Assoziationen - mit Anti-Intellektualismus und Anti-Elitismus z.B. gegen die EU, aber auch mit ausschließenden Forderungen gegenüber MigrantInnen und MuslimInnen. Diese Anschlussfähigkeit des Anti-Gender-Diskurses an andere kontroverse Diskussionen um eine Bedrohung von Identität macht „Gender“ zu einem „leeren Signifikanten“ (Laclau 1996, S. 36). Diese Funktion von „Gender“" erlaubt es wiederum Anti-Gender-Akteuren, mit je unterschiedlichen Anliegen anzuknüpfen und ihre Anliegen als Teil eines umfassenden Bedrohungsdiskurses zu reartikulieren.

Im „Anti-Genderismus“ konkretisiert sich damit - vergleichbar dem Antifeminismus an der Wende vom 19. zum 20. Jahrhundert (Weiss 2015) - eine anti-moderne und anti-pluralistische Haltung, die eine umfassende Bedrohung konstruiert, eine Bedrohung des Individuums, des Staates und der Gesellschaft. Gekämpft wird gegen die kulturrevolutionären Veränderungen der 1968er-Bewegungen, gegen Abtreibung, gegen Homosexualität, gegen den Wohlfahrtsstaat wie auch gegen Geflüchtete. Der Diskurs bleibt gewissermaßen unbegrenzt erweiterbar, da immer neue - oft nur sehr vage mit Geschlecht und Sexualität in Verbindung gebrachte - Themen adressiert werden können, die in der Perspektive der ganz unterschiedlichen Gruppierungen eine moralische, aber auch politische Wende, erfordern.

Geschlecht eignet sich deshalb so gut für eine gegenhegemoniale Mobilisierung, weil mit dem Bezug auf Geschlecht an Alltagserfahrungen angeknüpft werden kann - an die lang tradierte vermeintlich natürliche hierarchische Zweigeschlechtlichkeit und Heterosexualität, die durch Gleichstellungspolitik und Antidiskriminierungsrichtlinien zwar erschüttert, aber keineswegs erodiert sind, sondern noch immer die Lebensweisen und den Habitus der Menschen prägten. Der europäische AntiGender-Diskurs insbesondere rechtspopulistischer Akteure, aber in Koalition mit anderen Gruppen jenseits des rechten Spektrums spitzt somit die immer schon existierende Geschlechterungleichheit und den Sexismus kapitalistischer Gesellschaften und liberaler Demokratien zu. Umgekehrt moduliert Geschlecht aufgrund seiner binär gedachten Struktur antagonistisch-exkludierende Strategien. 
Anti-Gender-Mobilisierung ist somit funktional für ein radikal rechtes politisches Hegemonieprojekt. Im biopolitischen Arrangement der neuen Rechten - also im Versuch, neue Vorstellungen ,des Volkes“ durchzusetzen - nehmen Geschlechterund Sexualitätsverhältnisse eine prominente Rolle für die Verfugung eines national-populistischen autoritären Projekts ethnischer bzw. nationaler Homogenität und exkludierender Staatsbürgerschaft ein. Die Angriffe auf Feminismus und Gleichstellungspolitiken mit dem Label „Anti-Genderismus“ müssen also als Teil einer umfassenden Strategie begriffen werden, die für einen solchen politischen Umbau Konsens herzustellen sucht, die also autoritäre und ausschließende Diskurse und Praktiken zum common sense machen möchte.

Die diskursiven Logiken und Deutungsmuster fügen sich zu einem politischen Projekt, das den Kampf gegen kulturkritische und emanzipatorische Bewegungen seit den 1960er- und 1970er-Jahren zur Vertiefung ökonomischer und sozialer Ungleichheit und zur Propagierung eines nativistischen Volkskonstrukts nutzt. ,AntiGenderismus" dient daher den sich europaweit organisierenden autoritären rechtsextremen Kräften dazu, in ihrem Kampf um Hegemonie, Deutungsmacht und politische Macht die Grundsätze von Gleichberechtigung, Nichtdiskriminierung und Selbstbestimmung radikal in Frage zu stellen.

Die moralisch-politische Wende, die die (west-)europäischen Rechtspopulisten herbeizuführen trachten, zielt gegen eine ,sozial-demokratische“ Konstellation der Umverteilung und Integration, gegen neue Formen des partnerschaftlichen Zusammenlebens von Männern und Frauen, gegen Maßnahmen zur rechtlichen Gleichstellung von Frauen sowie gegen die Gleichstellung von LGBTIQ-Menschen. In einer als „Krise der Männlichkeit“ aufgebauschten Deutung soll dieses nationale Projekt auch Männlichkeit gegen ,die Eliten“ oder gegen „die Anderen“ mobilisieren und verstärken. Das rechtspopulistische Konzept des Volkes ist so an ethnopluralistische Konstrukte der Reinheit und der Ablehnung von Vermischungen (Schellenberg 2009, S. 2) anschließbar.

Unter Rückgriff auf eine biologisch-natürliche heterosexuelle Zweigeschlechtlichkeit lassen sich nämlich die Natürlichkeit wie auch Homogenität des Volkes symbolisieren (Diehl 2016, S. 17). Über die Geschlechtervorstellung ist ein nativistisches, biopolitisch-exklusives Volkskonstrukt vermittelbar, denn eine geschlechterideologisch begründete natürliche Zugehörigkeit rechtfertigt diskursiv den Ausschluss von „Anderen“ wie auch exklusive Formen nationaler Zugehörigkeit. Sowohl die FPÖ wie der Front National (jetzt: Rassemblement National) berufen sich auf eine distinkte heterosexuelle Zweigeschlechtlichkeit in ihren Volksvorstellungen. Auch die Intervention von Thilo Sarrazins (2010) Buch Deutschland schafft sich ab war ein Beispiel für eine maskulinistische biopolitische Anrufung, die Frauen die Rolle der Gebärerin zuschreibt, um ein ,gesundes“ und ,reines“ Volk argumentativ herzustellen (Sauer et al. 2017, S. 112).

Dieser ,autoritäre Populismus“ (Hall 1985, S. 116) strebt die Re-Naturalisierung gesellschaftlicher Ordnung als Legitimation dafür an, dass Demokratie im Sinne von Selbstbestimmung sich seit dem 2. Weltkrieg nicht nur als unmöglich erwiesen habe, sondern eben aufgrund der fundamentalen Ungleichheit von Menschen prinzipiell unmöglich sei. Demgegenüber entsteht die Idee einer autoritären Setzung des „Volkes“ insbesondere im exklusiven rechts-populistischen Sinne, eines Volkes, das 
nicht nur „natürlich“ homogen sei auf der Basis von pseudo-natürlicher Zweigeschlechtlichkeit und Heteronormativität, sondern das auch das betrogene Opfer von Eliten und Intellektuellen ist, somit schwach, passiv und handlungsunfähig ist und daher gerettet werden muss, Schutz und Führung braucht.

Rechtspopulistische Führungspersönlichkeiten imaginieren sich als diese Retter. Der Appell an das Volk als Souverän, die Forderung und das Versprechen direktdemokratischer Verfahren legen somit eher einen Bedarf an Führung jenseits denn Partizipation in repräsentativ-demokratischen Institutionen nahe. Aus der natürlichfamiliären und patriarchalen Vorstellung des Volkes folgt somit ein anti-demokratischer Gestus gegen die Selbstbestimmung des Volkes.

Geschlecht kann also im Anti-Gender-Diskursfeld zum Modulator eines umfassenden Kulturkampfes, eines Kampfes um politische Hegemonie werden. Rechtspopulistische Parteien wollen die Länder der EU im Sinne eines anti-liberalen, nationalautoritären bzw. national-sozialen Projekts umbauen (Balibar 2010).

Doch es deuten sich auch Spannungen und Widersprüche im Anti-Gender-Diskurs an. Die Thematisierung dieser Widersprüche, z. B. zwischen Klasse, Nationalität, Religion und Geschlecht, kann die sozialen Trennungen und Spaltungen reflektieren und möglicherweise für ein demokratisches Projekt nutzbar machen.

Funding Open access funding provided by University of Vienna.

Open Access Dieser Artikel wird unter der Creative Commons Namensnennung 4.0 International Lizenz (http://creativecommons.org/licenses/by/4.0/deed.de) veröffentlicht, welche die Nutzung, Vervielfältigung, Bearbeitung, Verbreitung und Wiedergabe in jeglichem Medium und Format erlaubt, sofern Sie den/die ursprünglichen Autor(en) und die Quelle ordnungsgemäß nennen, einen Link zur Creative Commons Lizenz beifügen und angeben, ob Änderungen vorgenommen wurden.

\section{Literatur}

AfD, 2013. Programm für die Bundestagswahl 2013. http://www.alternativefuer.de/programmhintergrund/programmatik/. Zugegriffen: 2. Mai 2015.

Amesberger, Helga, und Brigitte Halbmayr (Hrsg.). 2002. Rechtsextreme Parteien - eine mögliche Heimat für Frauen? Opladen: Leske \& Budrich.

Ausschuss für bürgerliche Freiheiten, Justiz und Inneres. 2014. Bericht über den EU-Fahrplan zur Bekämpfung von Homophobie und Diskriminierung aus Gründen der sexuellen Orientierung und der Geschlechtsidentität (2013/2183(INI)), A7-0009/2014. http://www.europarl.europa.eu/sides/getDoc. do?pubRef=-//EP//TEXT+REPORT+A7-2014-0009+0+DOC+XML+V0//DE. Zugegriffen: 2. Mai 2016.

Ausschuss für die Rechte der Frau und die Gleichstellung der Geschlechter. 2013. Bericht über sexuelle und reproduktive Gesundheit und die damit verbundenen Rechte (2013/2040(INI)), A70426/2013. http://www.europarl.europa.eu/sides/getDoc.do?pubRef=-//EP//TEXT+REPORT+A72013-0426+0+DOC+XML+V0//DE. Zugegriffen: 2. Mai 2016.

Balibar, Etienne. 2010. Kommunismus und (Staats)Bürgerschaft. Überlegungen zur emanzipatorischen Politik. In Das Staatsverständnis von Nicos Poulantzas. Der Staat als gesellschaftliches Verhältnis, Hrsg. Alex Demirovic, Stephan Adolphs, und Serhat Karakayali, 19-33. Baden-Baden: Nomos.

de Beauvoir, Simone. 1992. Das andere Geschlecht. Sitte und Sexus der Frau. Reinbek: Rowohlt.

Blum, Alice. 2015. Germany. In Gender as symbolic glue. The position and role of far right parties in the anti-gender mobilizations in Europe, Hrsg. Esther Kováts, Maari Pōim, 40-61. Budapest: Foundation for European Progressive Studies.

Brustier, Gaël. 2015. France. In Gender as symbolic glue. The position and role of far right parties in the anti-gender mobilizations in Europe, Hrsg. Esther Kováts, Maari Pōim, 19-39. Budapest: Foundation for European Progressive Studies.

Camus, Renaud. 2016. Revolte gegen den großen Austausch. Schnellroda: Antaios. 
Candeias, Mario. 2016. Gegenmittel. Autoritärer Neoliberalismus und Rechtspopulismus. Perspektiven einer verbindenden linken Partei. In Transformation der Demokratie - demokratische Transformation, Hrsg. Alex Demirovic, 9-34. Münster: Westfälisches Dampfboot.

Chołuj, Bożena. 2015. „Gender-Ideologie“ - ein Schlüsselbegriff des polnischen Anti-Genderismus. In Anti-Genderismus. Sexualität und Geschlecht als Schauplätze aktueller politischer Auseinandersetzungen, Hrsg. Sabine Hark, Paula-Irene Villa, 219-237. Bielefeld: transcript.

Connell, Raewyn, und James W. Messerschmidt. 2005. Hegemonic masculinity. Rethinking the concept. Gender \& Society 19(6):829-859.

Diehl, Paula. 2016. Demokratische Repräsentation und ihre Krise. Aus Politik und Zeitgeschichte 66(40-42):12-17.

Dietze, Gabriele. 2015. Anti-Genderismus intersektional lesen. Zeitschrift für Medienwissenschaft 13(2):125-127.

Esping-Andersen, Gosta. 1990. Three worlds of welfare capitalism. Cambridge: Polity Press.

Farris, Sara R. 2017. In the name of women's rights. The rise of femonationalism. Durham, London: Duke University Press.

Favier, Anthony. 2015. Catholics and gender. A historical approach. http://www.booksandideas.net/IMG/ pdf/catholiques_genre_en_relu_final.pdf. Zugegriffen: 25. Apr. 2016.

Ganz, Kathrin. 2015. PEGIDA gegen den Gender-Wahn: Rechte Mobilisierung und die Krise sozialer Reproduktion. Blog Feministische Studien. http://blog.feministische-studien.de/2015/02/pegida-gegenden-gender-wahn-rechte-mobilisierung-und-die-krise-sozialer-reproduktion/. Zugegriffen: 13. Apr. 2016.

Graff, Agnieszka. 2014. Report form the gender trenches: War against "genderism" in Poland. European Journal of Women's Studies 21(4):431-442.

Grzebalska, Weronika. 2016. Why the war on "gender ideology" matters—and not just to feminists. Antigenderism and the crisis of neoliberal democracy. Visegrad/Insight. http://visegradinsight.eu/whythe-war-on-gender-ideology-matters-and-not-just-to-feminists/. Zugegriffen: 15. Apr. 2019.

Hall, Stuart. 1985. Authoritarian populism: a reply to Jessop et al. New Left Review I/151:115-124.

Hark, Sabine, und Paula-Irene Villa. 2015. „Anti-Genderismus“ - Warum dieses Buch? In Anti-Genderismus. Sexualität und Geschlecht als Schauplätze aktueller politischer Auseinandersetzungen, Hrsg. Sabine Hark, Paula-Irene Villa, 7-13. Bielefeld: transcript.

Hodzić, Amir, und Nataša Bijelić. 2014. Neo-Conservative Threats to Sexual and Reproductive Health \& Rights in the European Union, CESI. http://www.cesi.hr/attach/_n/neo-conservative_threats_to_ srhr_in_eu.pdf. Zugegriffen: 30. Apr. 2019.

Kimmel, Michael. 2013. Angry white men. American Masculinity at the End of an Era. New York: Bold Type Books.

Korolczuk, Elżbieta. 2015. „the war on gender“ from a transnational perspective-lessons for feminist strategising. In Anti-gender movements on the rise? Strategising for gender equality in central and eastern europe, 43-53. Berlin:: Heinrich Böll Stiftung.

Kuby, Gabriele. 2006. Die Gender Revolution - Relativismus in Aktion. Kisslegg: fe-medienvlg.

Kuhar, Roman. 2015. Playing with science: sexual citizenship and the Roman catholic church counternarratives in Slovenia and Croatia. Women's Studies International Forum 49:84-92.

Laclau, Ernesto. 1996. Emancipation(s). London/New York: Verso.

Laclau, Ernesto, und Chantal Mouffe. 2001. Hegemony and Socialist Strategy. Towards a Radical Democratic Politics. London, New York: Verso.

Lang, Juliane. 2015. Familie und Vaterland in der Krise. Der extrem rechte Diskurs um Gender. In AntiGenderismus. Sexualität und Geschlecht als Schauplätze aktueller politischer Auseinandersetzungen, Hrsg. Sabine Hark, Paula-Irene Villa, 167-181. Bielefeld: transcript.

Langan, Mary, und Ilona Ostner. 1991. Geschlechterpolitik im Wohlfahrtsstaat. Aspekte im internationalen Vergleich. Kritische Justiz 24(3):302-317.

de Lange, Sarah, und Liza M. Mügge. 2015. Gender and right-wing populism in the Low Countries: ideological variations across parties and time. Patterns of Prejudice 49(1):61-80.

Mad'arová, Zuzana. 2015. Love and fear. Argumentative strategies against gender equality in Slovakia. In Anti-gender movements on the rise? Strategising for gender equality in central and Eastern Europe, 33-42. Berlin: Heinrich Böll Stiftung.

Marschütz, Gerhard. 2014. Trojanisches Pferd Gender? Theologische Anmerkungen zur jüngeren Genderdebatte im katholischen Bereich. In Aus Liebe zu Gott - im Dienst an den Menschen: Spirituelle, pastorale und ökumenische Dimensionen der Moraltheologie, Hrsg. Kerstin Schlögel, Gunter M. PrüllerJagenteufel, 432-454. Münster: Aschendorff. 
Mayer, Stefanie, und Birgit Sauer. 2017. "Gender Ideology" in Austria: Coalitions around an Empty Signifier. In Anti-Gender Campaigns in Europe. Mobilizing against Equality, Hrsg. Roman Kuhar, David Paternotte, 19-30. London: Rowman and Littlefield.

Mayer, Stefanie, Iztok Sori, und Birgit Sauer. 2015. Gendering "the people": heteronormativity and "ethnomasochism" in populist imaginery. In Populism, Media and Education. Challenging discrimination in contemporary digital societies, Hrsg. Maria Ranieri, 84-104. London, New York: Routledge.

Moss, Kevin. 2017. Gender ideology and geopolitics: the role of Russia. In Anti-gender campaigns in Europe. Mobilizing against equality, Hrsg. Roman Kuhar, David Paternotte, 195-213. London: Rowman and Littlefield.

Mudde, Cas, und Cristobal R. Kaltwasser. 2015. Vox Populi or vox masculini? Populism and gender in Northern Europe and South America. Patterns of Prejudice 49(1):16-36.

Päpstlicher Rat für die Familie. 2000. Ehe, Familie und „faktische Lebensgemeinschaften“. http:// www.vatican.va/roman_curia/pontifical_councils/family/documents/rc_pc_family_doc_20001109_ de-facto-unions_ge.html. Zugegriffen: 28. Apr. 2016.

Paternotte, David. 2014. Christian trouble: the catholic church and the subversion of gender. http:// councilforeuropeanstudies.org/critcom/christian-trouble-the-catholic-church-and-the-subversionof-gender/. Zugegriffen: 15. Apr. 2016.

Paternotte, David. 2015. Blessing the crowds. Catholic mobilisations against gender in Europe. In AntiGenderismus. Sexualität und Geschlecht als Schauplätze aktueller politischer Auseinandersetzungen, Hrsg. Sabine Hark, Paula-Irene Villa, 129-147. Bielefeld: transcript.

Pfaller, Robert. 2017. Erwachsenensprache: Über ihr Verschwinden aus Politik und Kultur. Frankfurt/M.: Fischer.

Radhakrishnan, Smitha, und Cinzia Solari. 2015. Empowered women, failed patriarchs: neoliberalism and global gender anxieties. In Sociology Compass 9(9):784-802.

Rosenkranz, Barbara. 2008. MenschInnen. Gender-Mainstreaming. Auf dem Weg zum geschlechtslosen Menschen. Graz: Ares.

Sarrazin, Thilo. 2010. Deutschland schafft sich ab. Wie wir unser Land aufs Spiel setzen. München: Deutsche Verlags-Anstalt.

Sauer, Birgit, Roman Kuhar, Edma Ajanović, und Aino Saarinen. 2017. Exclusive intersections: constructions of gender and sexuality. In Understanding the populist shift. Othering in a Europe in crisis, Hrsg. Gabriella Lazaridis, Giovanna Campani, 104-121. London/New York: Routledge.

Schellenberg, Britta. 2009. Aktuelle Entwicklungen im europäischen Rechtsextremismus. http://www. bpb.de/politik/extremismus/rechtsextremismus/41221/analyse-rechtsradikale-in-europa. Zugegriffen: 06.2016.

Schmincke, Imke. 2015. Das Kind als Chiffre politischer Auseinandersetzungen am Beispiel neuer konservativer Protestbewegungen in Frankreich und Deutschland. In Anti-Genderismus. Sexualität und Geschlecht als Schauplätze aktueller politischer Auseinandersetzungen, Hrsg. Sabine Hark, PaulaIrene Villa, 93-107. Bielefeld: transcript.

Siim, Birte, und Susi Meret. 2019. Dilemmas of citizenship and evolving civic activism in Denmark. In Citizen's activism and solidarity movements. Contending with populism, Hrsg. Birte Siim, Anna Krasteva, und Aino Saarinen, 25-50. Basingstoke: Palgrave Macmillan.

Stambolis-Ruhstorfer, Michael, und Josselin Tricou. 2017. Resisting "gender theory" in France: a fulcurm for religious action in a secular society. In Anti-Gender Campaigns in Europe. Mobilizing against Equality, Hrsg. Roman Kuhar, David Paternotte, 79-97. London: Rowman and Littlefield.

Weiss, Alexandra. 2013. „Geschlechterkampf“ - Inszenierungen von Frauenmacht und Männerleid. In Geschlechterverhältnisse und neuе Öffentlichkeiten. Feministische Perspektiven, Hrsg. Birgit Riegraf, Hanna Hacker, Heike Kahlert, Brigitte Liebig, Martina Peitz, und Rosa Reitsamer, 37-57. Münster: Westfälisches Dampfboot.

Weiss, Alexandra. 2015. Gefährdete Positionen. Antifeminismus als Reaktion auf Krise und Transformation. In derdiedas bildende, 3(5), o.S. Wien: akademie der bildenden künste

Wimbauer, Christine, Mona Motakef, und Julia Teschlade. 2015. Prekäre Selbstverständlichkeiten. Neun prekarisierungstheoretische Thesen zu Diskursen gegen Gleichstellungspolitik und Geschlechterforschung. In Anti-Genderismus. Sexualität und Geschlecht als Schauplätze aktueller politischer Auseinandersetzungen, Hrsg. Sabine Hark, Paula-Irene Villa, 41-57. Bielefeld: transcript. 